\title{
2017 Wilderness E Environmental Medicine Peer Reviewers
}

The Editor-in-Chief and Managing Editor express their sincere appreciation to the following individuals who performed peer reviews for articles submitted to Wilderness \& Environmental Medicine. WEM continues to have a growing impact on the scientific community. Our peer reviewers play an essential role in ensuring the merit and quality of the manuscripts we publish. Many of these individuals reviewed multiple papers, and some also serve on the Editorial Board and maintain Section and/or Associate Editor duties. Without their service we could not produce this high-quality medical journal.

(Note: * indicates WEM Editorial Board membership; † indicates WEM Reviewer-in-Training.)

William Adams, USA

John Anderson, USA

Paul Auerbach, USA

Howard Backer, USA*

Buddha Basnyat, Nepal*

Brad Bennett, USA*

Michael Bergeron, USA

Claire Berryman, USA

James Beverly, USA

Cindy Bitter, USA

Michael Bogdasarian, USA

Gemma Bornick, USA

Ralph Bovard, USA

Benjamin Brackett, USA ${ }^{\dagger}$

Graham Brant-Zawadzki, USA ${ }^{\dagger}$

Aaron Brillhart, USA

Julio Brito, Chile

Monika Brodmann Maeder, Switzerland

Omer Burnett, USA

Martin Burtscher, Austria

Emilie Calvello Hynes, USA

Aaron Campbell, USA*

Michael Cardwell, USA

Elizabeth Carlton, USA

Jim Caruso, USA

Michael Caudell, USA

Jean-Philippe Chippaux, France

Kirsten Coffman, USA

Anita Cote, Canada

Cord Cunningham, USA

Tracy Cushing, USA*

Michael Dacre, USA

Christopher Davis, USA

Luc De Haro, France

Leonardo De Sousa, Venezuela

Juan Del Coso, Spain

Thomas Deloughery, USA

Robert W. Derlet, USA
James Diaz, USA

Alberto Dolci, France

Steve Donelan, USA*

Jennifer Dow, USA

Brendon Drew, USA

Gerald Dubowitz, USA

Michael Duchene, Austria

Ola Halina Dunin-Bell, Canada

Kurt Eifling, USA

John Ellerton, UK

Emmanuel Cauchy, France

Timothy B. Erickson, USA*

Preston Fedor, USA

Andrea Ferrari, USA ${ }^{\dagger}$

Andrew Fock, Australia

Joseph Forrester, USA

Luanne Freer, USA

Maria Gadelha, Brazil

Samuel Galvagno, USA

Flavio Gaudio, USA

Manuel Genswein, Switzerland

Charles Gerardo, USA

Gordon Giesbrecht, Canada

Jeff Godin, USA

Todd Golden, USA

Petra Golja, Slovenia

Torrey Goodman, USA

Mike Greene, UK

Stefano Grigolato, Italy

Colin Grissom, USA*

Leah Groves, USA

A. Guerrero, USA

Tracy Høeg, USA

Peter Hackett, USA*

Vidal Haddad, Jr., Brazil

Mark Harper, UK

N. Stuart Harris, USA

Richard Harris, Australia

Michael Hauty, USA
Tatiana Havryliuk, USA

Seth Hawkins, USA

Alana Hawley, USA

Carlton Heine, USA

Mary Hessert, USA

David Hillebrandt, UK

Alastair Hodges, Canada

Christopher Holstege, USA

Chris Imray, UK

Hillary Irons, USA

Arthur (Tony) Anthony Islas, USA

Katherine James, USA

Ramin Jamshidi, USA

James Jeng, USA

Eric Johnson, USA

Barbara Jones, USA

Noah Jordahl, USA

Lee Kaplan, USA

Al Katz, USA

Bengt Kayser, Switzerland

Robert Kenefick, USA*

Linda Keyes, USA

Morteza Khodaee, USA

Jenevieve Kincaid, USA ${ }^{\dagger}$

Michelle King, USA

Kenneth Kizer, USA*

Joseph Kotora, USA

Brian Krabak, USA

Jordan Lane, USA ${ }^{\dagger}$

Ricky Langley, USA

Justin Lawley, USA

Wolfgang Lederer, Austria

Jay Lemery, USA*

Richard Levitan, USA

Maureen Lichtveld, USA

James Lieberman, USA

Grant Lipman, USA*

Matthew Lippi, USA ${ }^{\dagger}$ 
Andrew Luks, USA

Darryl Macias, USA*

Tom Mallinson, UK

Laura McGladrey, USA ${ }^{\dagger}$

Todd McGrath, USA

Scott McIntosh, USA

Igor Mekjavic, Slovenia

John Meyers, USA

Brendan Milliner, USA ${ }^{\dagger}$

Simon Mitchell, New Zealand

Wuelton Monteiro, Brazil

Patrick Morgan, UK

Maria Nemethy, USA

Benjamin Nicholson, USA

Matias Nochetto, USA

Robert Norris, USA

Gerald O'Malley, USA

Edward J. (Mel) Otten, USA*

Peter Paal, UK

Prativa Pandey, Nepal

Deb Pandey, Nepal

Stefan Pasiakos, USA

Lara Philips, USA

Kim Prisk, USA

Matiram Pun, Nepal*

Robert Quinn, USA*

Jeremiah Ray, USA

Peter Rhee, USA
Paul Richards, UK

Rosemary Rochford, USA

George Rodway, USA*

Terry Rolan, USA

Steven Roy, Canada

Brent Ruby, USA

Anne-Michelle Ruha, USA

David Rust, USA

Emily Sagalyn, USA

Renee Salas, USA

Volker Schöffl, Germany*

Tod Schimelpfenig, USA

Andrew Schmidt, USA

Robert Schoene, USA

Walter Schrading, USA

Zina Semenovskaya, USA

Suzanne Shepherd, USA

Jessica Slim, USA

William Smith, USA

Cecilia Sorensen, USA

Susanne Spano, USA

Cynthia Stevens, USA

Giacomo Strapazzon, Italy

Gage Stuntz, USA ${ }^{\dagger}$

Douglas Sward, USA

Choo Hock Tan, Malaysia

David Tan, USA

Kathrine Tan, USA
Christopher Tedeschi, USA

Martha Tissot van Patot, USA

Suha Turkmen, Turkey

Lise Van Susteren, USA

Christopher Van Tilburg, USA

Stefano Veraldi, Italy

Arpana Vibhuti, India

Jenny Visser, New Zealand

Scott Votey, USA

David Warrell, UK

Ian Wedmore, USA

Lori Weichenthal, USA

Scott Weinstein, Australia*

Jenna White, USA

Julian White, Australia

Eranga Wijewickrama, Sri Lanka

Peter Wilmshurst, UK

Bryan Wilson, USA

Matthew Wilson, USA

Jeremy S. Windsor, UK*

Kenneth Winkel, Australia

Dean Winslow, USA

Justin Wright, USA

Scott Young, USA

Ken Zafren, USA*

Liming Zhang, China

Keith Zirkle, USA 